\title{
Interaction of Novel Metal Complexes with DNA: Synthetic and Structural Aspects
}

\author{
Bernhard Spingler*a, Chiara Da Pieve ${ }^{a}$, Alfredo Medina-Molnera, Philipp M. Antonia ${ }^{\text {, }}$, and \\ Maria Grazia Santangelob
}

\begin{abstract}
Metal ions bind to nucleic acids at various positions. This binding can be modulated by using metal complexes with appropriate ligands. Novel mono- and especially dinuclear metal complexes could be a powerful tool to detect rare, but still physiologically relevant, forms of DNA, e.g. the left-handed Z-DNA. In this review, our recent research activities in this area of bioinorganic chemistry are summarized. A special emphasis is laid on the synthetic challenges that arose upon the synthesis of the polyamine ligands. Further, some rather unusual approaches to elucidate the solution structure of copper bound to guanosine monophosphate with the help of pulsed EPR techniques like ENDOR and HYSCORE are described.
\end{abstract}

Keywords: Bioinorganic chemistry · Crystal structures - Mono- and Dinuclear Metal Complexes · Synthetic Chemistry $\cdot$ Z-DNA

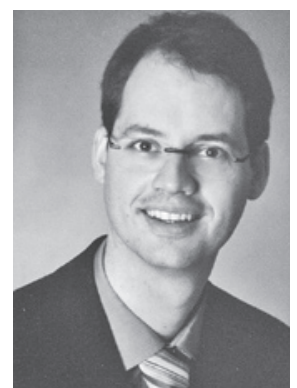

Bernhard Spingler was born in July 1972 in Basel, Switzerland. In 1991, he started studying chemistry at the University of Basel. He received his doctoral degree in 1998 (summa cum laude), working in the field of asymmetric organic chemistry. For two years, he joined the group of Stephen J. Lippard as postdoctoral fellow at MIT, Cambridge, USA, where he worked on the interaction of anti-cancer drugs with DNA. At the University of Zurich, Bernhard Spingler started his independent career in the field of bioinorganic chemistry.

\footnotetext{
${ }^{\star}$ Correspondence: Dr. B. Spingler ${ }^{a}$

Tel.: + 41446354656

Fax: + 41446356803

E-mail: spingler@aci.uzh.ch

alnstitute of Inorganic Chemistry

University of Zurich

Winterthurerstr. 190

$\mathrm{CH}-8057$ Zürich

'Laboratory of Physical Chemistry

ETH Zürich

$\mathrm{CH}-8093$ Zürich
}

\section{Introduction}

The study of the interactions of metal complexes with oligonucleotides has always been at the centre of bioinorganic chemistry. ${ }^{[1]}$ Nucleobases, phosphate esters and, even sugars, all offer various atoms that can coordinate to the metal centre in cases of inner sphere binding. ${ }^{[2]}$ On the other hand, metal complexes with ligands that contain extended aromatic moieties can bind either to the grooves of double-stranded oligonucleotides or intercalate between the base pairs. In this second case, there is only an indirect interaction between metal centre and oligonucleotides. If the positive charge of the metal has not completely been compensated by anionic ligands, this interaction can be electrostatic and is therefore isotropic. In the case of polyaromatic ligands, the role of the metal centre can be mainly a structural one, by maintaining a certain geometry, either between several ligands coordinating to the same metal or by fixing one spatial arrangement of an otherwise flexible ligand.

We approach this research topic from a synthetic side. Metal complexes with an expected property towards DNA are synthesized and then their properties tested. We are especially interested in studying the interaction of metal complexes with left-handed DNA, also called Z-DNA. This particular high-energy form of DNA can only be formed with alternating purinepyrimidine sequences. Nevertheless, such sequences are found in our genes. The induction of Z-DNA requires, additionally, the presence of polycations or high salt concentrations. The temporary formation of Z-DNA has been correlated with the transcription of gene sequences that fulfil the above-mentioned criteria.[3] Therefore, Z-DNA levels are elevated in rapidly proliferating cells. This fact is our main motivation to study metal complexes with the final goal of finding a Z-DNA selective reagent that can be used in cells. Some of our earlier work ${ }^{[4]}$ in this direction was discussed in this journal in 2005. ${ }^{[5]}$ Here, we are presenting our recent progress in the context of the actual literature.

\section{Recent Research}

The metal complex 1a (Fig. 1: 1, $\mathrm{M}=$ $\mathrm{Ni}, \mathrm{R}^{1}=\mathrm{F}$ ) has been reported to induce the transition of B- to Z-DNA. ${ }^{[6]}$ We were interested in exploring the effect of the metal (copper and zinc instead of nickel) and the substituents $\mathrm{R}^{1}$ and $\mathrm{R}^{2}$ upon the efficiency of the complexes $\mathbf{1}$ to induce Z-DNA.[4] Therefore, we needed a practicable synthetic access to the ligand type $\mathbf{2}$. For the simplest variant of $\mathbf{2}, \mathbf{2} \mathbf{a}\left(\mathrm{R}^{1}, \mathrm{R}^{2}=\mathrm{H}\right)$, we followed the previously reported procedure, but the product could be isolated in

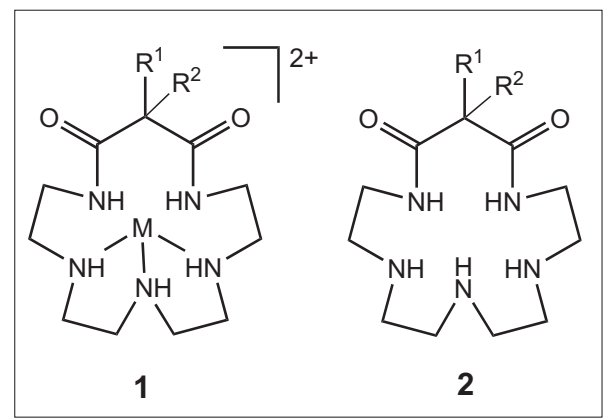

Fig. 1. Ligands 2 and the corresponding mononuclear metal complexes 1 used for the induction of the left-handed Z-DNA. 


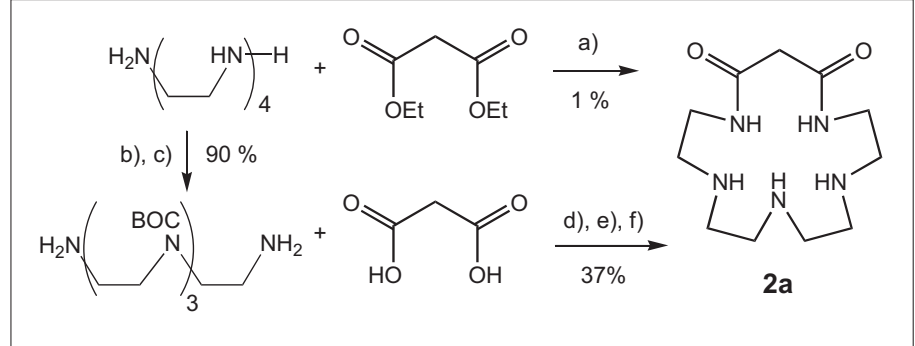

Scheme 1. Two paths leading to 2a: a) following the literature: $57 \mathrm{mM}$, 10 days; b) 4.25 equiv. $\mathrm{CF}_{3}$ COOEt; c) 5 equiv. BOC; d) $4 \mathrm{mM}$ of amine, 2 equiv. HOBt, 3 equiv. DCC; e) 20 equiv. TFA; f) anion exchange column.

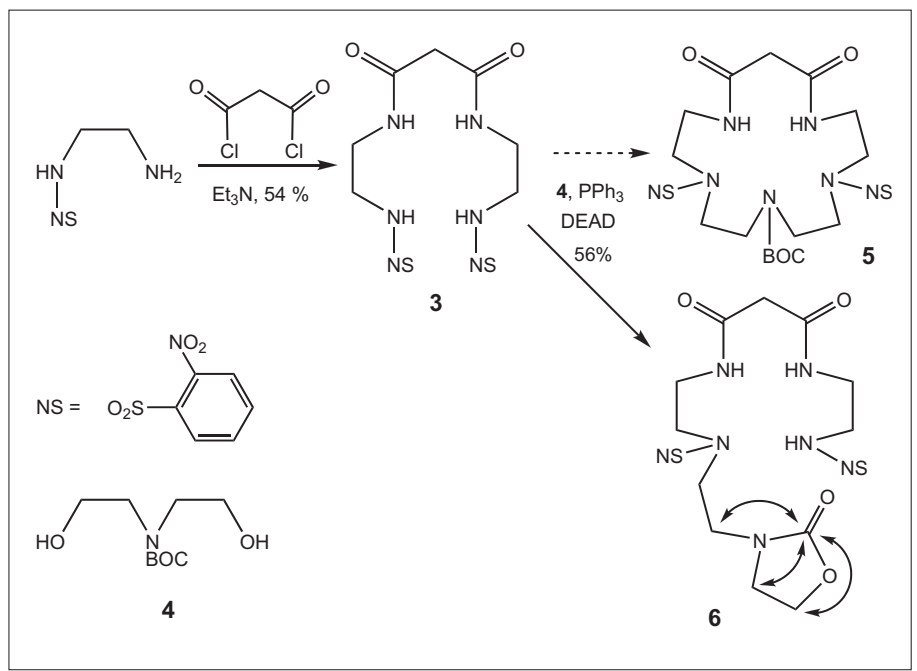

Scheme 2. Attempted synthesis of $\mathbf{5}$ that yielded the acyclic compound 6 instead. Arrows in the drawing of $\mathbf{6}$ mark the cross peaks found in a long-range $\left[{ }^{1} \mathrm{H},{ }^{13} \mathrm{C}\right]$ HMBC NMR spectrum.



Scheme 3. Initially proposed retrosynthetic scheme for the dinucleating ligand 7.

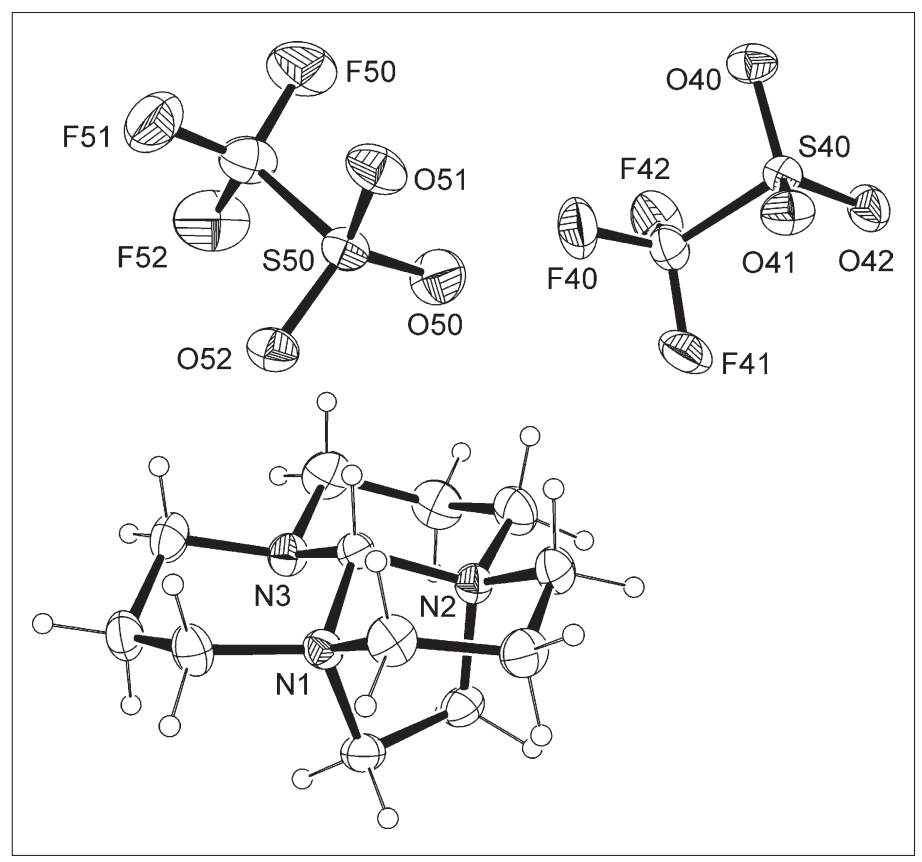

Fig 2. ORTEP presentation drawn at $30 \%$ probability of one of the two molecules in the asymmetric unit of $\mathbf{9}$. only $1 \%$ yield compared with the reported yield of $15 \%$ (reaction a in Scheme 1 ). Therefore, several approaches to $\mathbf{2 a}$ were tested, finally a classical strategy using various protecting groups turned out to be the only practicable access to the ligand (reactions b-f in Scheme 1). [7] Instead of trifluoroacetate, we have also employed 1-(4,4-dimethyl-2,6-dioxocyclohexylidene)ethyl (DDE) as a protecting group for the primary amines. The disadvantages of the DDE route were a slightly lower yield (77\% versus $90 \%$ ) and the need for an additional column chromatography. ${ }^{[7]}$

As another approach to $\mathbf{2 a}$, we have also tried a double Mitsunobu reaction to form the protected azamacrocycle $\mathbf{5}$ (Scheme 2). ${ }^{[8]}$ Successful cases of Mitsunobu reactions to form medium-sized cycles have been reported. ${ }^{[9]}$ Analysis of our reaction revealed that the first Mitsunobu reaction had worked, however the second alcohol had attacked the carbonyl group of the BOC protecting group to yield a 1,3-oxazolidin-2-one. We found in the literature one report of a similar case where a six-membered cyclic carbamate formed in the presence of triphenylphosphine. ${ }^{[10]}$ In a review about side reactions involving the BOC protecting group, this particular reactivity was explained with the formation of an alkoxyphosphonium salt.[11] Unfortunately, when diethanolamine, 1,1'-(azodicarbonyl)dipiperidine (ADDP) and tri- $n$-butyl phosphine (TBP) ${ }^{[12]}$ were reacted together with $\mathbf{3}$, only the starting materials could be isolated.

Another synthetic challenge was faced when we wanted to synthesize alkylenebridged 1,5,9-triazacyclodecane dinucleating ligands 7 (Scheme 3 ). The propylene-bridged ligand $\mathbf{7 b}(\mathbf{7}, \mathrm{n}=2)$ was already known from the literature and its synthesis posed no problems at all.[13] However, the synthesis of the corresponding ethylene-bridged ligand $7 \mathbf{a}(7, \mathrm{n}=1)$ was not so straightforward. Initially, the ${ }^{1} \mathrm{H}-\mathrm{NMR}$ spectrum of the reaction product of 1,5,9-triazatricyclo[7.3.1.0]tridecane 8 with 1,2-ethylene glycol bis(triflate) remained mysterious. Crystals could be grown that were suitable for single crystal X-ray analysis (Fig. 2). The product turned out to be the result of a double alkylation to form the unknown tetracylic diammonium cation 9.[14] No reaction was observed when 1,5,9-triazatricyclo[7.3.1.0] tridecane 8 was allow to react with 1,2-dibromoethane, even in the presence of two equivalents of potassium iodide. The tar- geted compound $\mathbf{7 b}$ could finally be synthesized using oxalyl chloride together with 8. The corresponding diamide was reduced with lithium aluminium hydride and treatment with triflouromethanesulfonic acid yielded the biscycle $\mathbf{7 b}$, as the triflate salt (Scheme 4).

When studying the effect of copper complexes $\mathbf{1}\left(\mathrm{R}^{1}=\mathrm{F}, \mathrm{R}^{2}=\mathrm{H}, \mathrm{CH}_{3}, \mathrm{~F}\right)$, a central question arose: where do these complexes bind to the (Z-)DNA? A collaboration was started with the group of the late Prof. Arthur Schweiger at the ETH. To our surprise, there were no reports of pulsed EPR studies even on the simplest possible system: copper and 5'-guanosine monophosphate. Therefore, we began to explore this model system with the help of various sophisticated EPR techniques, like Mims and Davies ENDOR as well as HYSCORE.[15] In the end, we could not only qualitatively map the coordination environment of the copper but also determine some essential distances between the copper centre and selected atoms of the guanonsine monophosphate. Continuous-wave EPR showed unambiguously a copper(II) in a $\left(\mathrm{d}_{\mathrm{x}^{2}-\mathrm{y}^{2}}{ }^{1}\right)$ ground state. The HYSCORE spectrum presents at first glance two types of weakly coordinated hydrogen atoms (Fig. 3a). The 


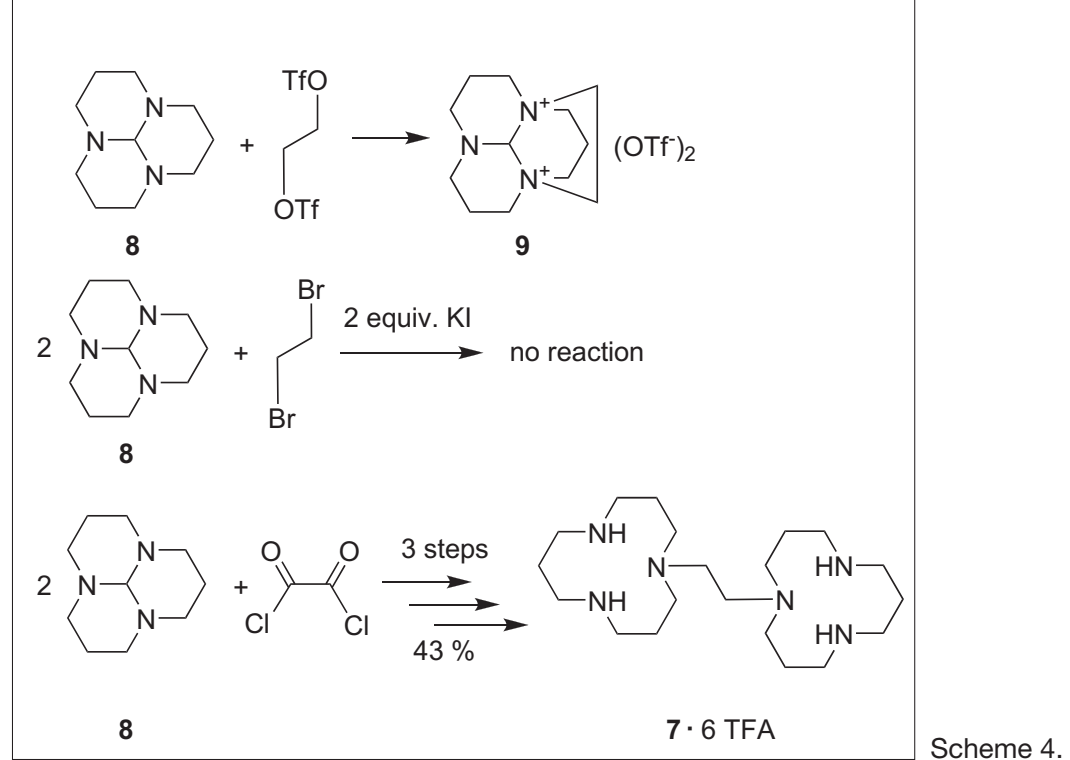

first set was assigned to protons of either axially coordinated molecules or close-by solvent molecules as previously done in the literature. ${ }^{[16]}$ The other peaks could be assigned to equatorially coordinated water a)

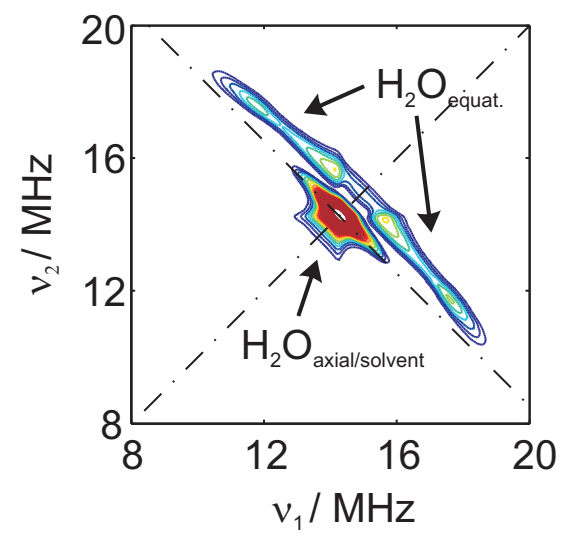

c)



b)

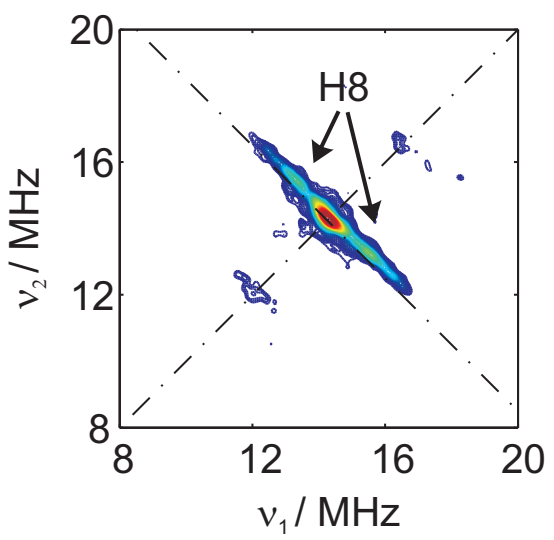

d)

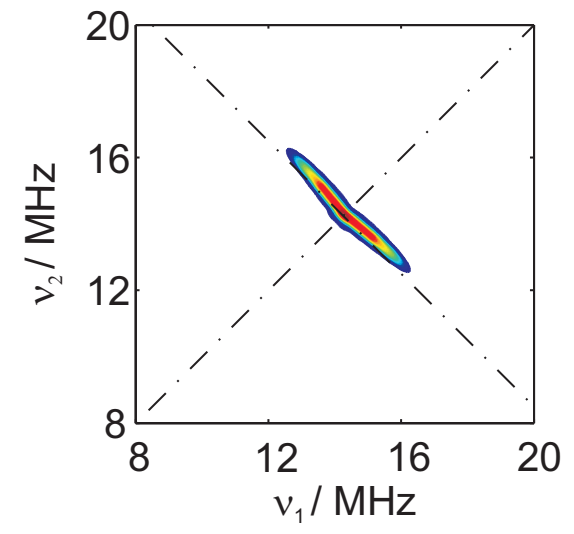

Fig. 3. X-band HYSCORE EPR spectra (above) and simulations (below). a),c) Cu(II)-5'-GMP; b),d) $\mathrm{Cu}(\mathrm{II})-5$ '-GMP in deuterated solvents. This Fig. was taken and adapted with kind permission from Springer Science+Business Media: Journal of Biological Chemistry, Structural analysis of Cu(॥) ligation to the 5'-GMP nucleotide by pulse EPR spectroscopy, 12, 2007, 772, Fig. 4. [15]

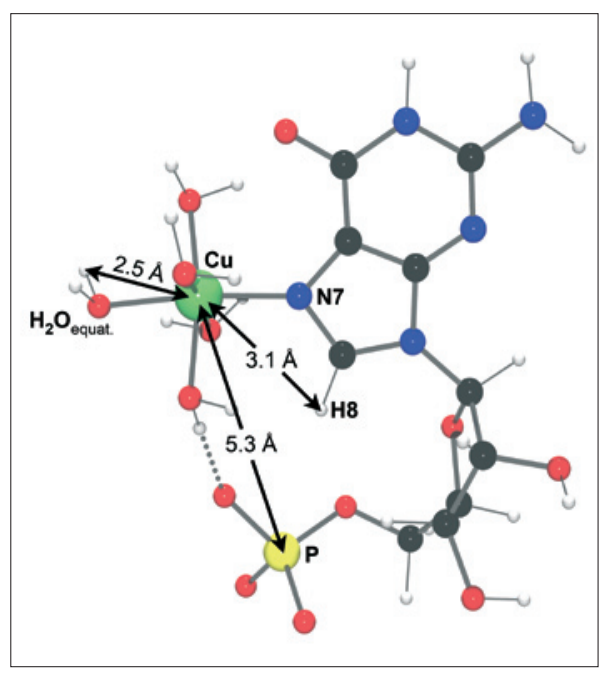

Fig. 4. Model of Cu(II)-5'-GMP as suggested by pulsed EPR techniques. Measured distances and used technique: $\mathrm{Cu}-\mathrm{H}\left(\mathrm{H}_{2} \mathrm{O}_{\text {equato }}\right)$ : $2.5 \pm 0.1 \AA ̊$ by HYSCORE, $\mathrm{Cu}-\mathrm{H}(8): 3.1 \pm 0.1 \AA$ by HYSCORE, Cu-P: $5.3 \pm 0.2 \AA \AA$ by Mims ENDOR.

EPR can be used like the NOE technique in NMR to determine distances between selected atoms. The use of 5'-GMP with all the exchangeable protons deuterated in deuterated solvents allowed detection of the proton peaks of $\mathrm{H}(8)$ (Fig. 3b). Again the distance between the copper and now $\mathrm{H}(8)$ could be determined. A model resulting from all the measurements undertaken is shown in Fig. 4. The copper is equatorially coordinated by water molecules and one guanosine monophosphate via the $\mathrm{N}(7)$ (O3N coordination). Mims ENDOR spectrum finally detected a weak coupling between the copper and the phosphorous of the phosphate group. From the coupling constant, a distance of $5.3 \pm 0.2 \AA$ could be calculated. This is in line with a single hydrogen bridge that links the copper via one coordinated water molecule to the phosphate. Such an arrangement has been found before in the crystalline state. ${ }^{[17]}$ Our studies are the first proof for the existence of such an arrangement in (frozen) solution. Previous models of macrochelates of metal purine 5' -monophosphate always assumed a direct coordination of the metal to the phosphate. ${ }^{[18]}$

In Z-DNA, the N(7) atoms of the guanine nucleobases are exposed to the solvent. We thought that sterically demanding dinuclear complexes might lead to a stronger and more selective interaction to the Z-DNA than the analogue mononuclear complexes. Inspired by a dinuclear tetrapyrazoyl complex, ${ }^{[19]}$ we studied the induction of Z-DNA by the novel dinuclear complex 10 (Fig. 5).[20] The complex induced Z-DNA at half the metal concentration than the corresponding mononuclear complex. However, the in situ prepared complex 11 consisting of the ligand and one equivalent of nickel induced Z-DNA 




10

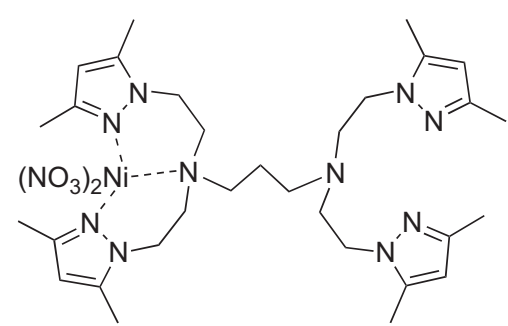

11
Fig. 5.

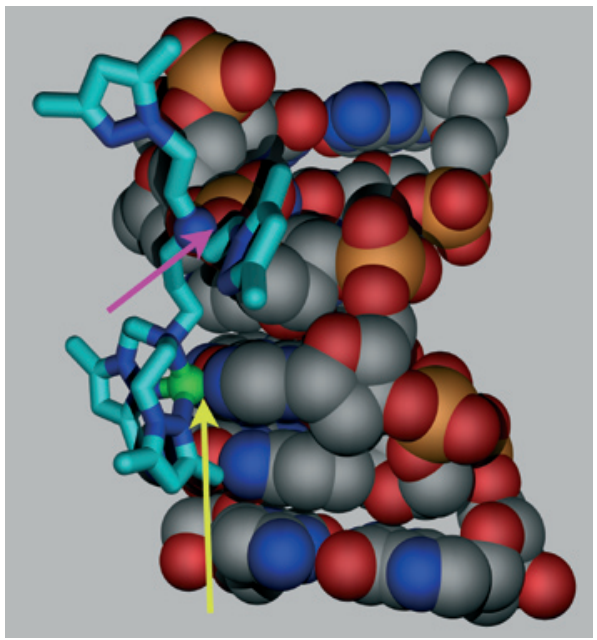

Fig. 6. Model of the interaction between protonated 11 and Z-DNA. The carbon atoms of $\mathbf{1 1}$ are shown in light blue. Coordination of the nickel atom to $N(7)$ is highlighted with a yellow arrow, the hydrogen bond between the protonated ternary nitrogen and the phosphate group with a purple arrow.

equally well. In the aqueous environment at $\mathrm{pH}$ 7, the free ternary nitrogen of $\mathbf{1 1}$ will be protonated. Therefore we conclude that the active species is $\mathbf{1 1}$ with an additional proton at the aliphatic nitrogen. A model of how this protonated $\mathbf{1 1}$ might interact with Z-DNA is shown in Fig. 6. The metal coordinates to $\mathrm{N}(7)$ of guanosine, the protonated nitrogen forms a hydrogen bond of the same strand.

\section{Ongoing Work}

One topic we are currently working on is the recognition of Z-DNA. Balaz et al. reported in 2005 the recognition of Z DNA by the zinc(II) complex of 5,10,15,20-tetrakis(4-N-methylpyridinium)porphyrin. [21] The zinc porphyrin was added to a previously induced Z-DNA form of poly d(GC) and a $C D$ signal in the visible range recorded. We reproduced these experiments with porphyrins that contained different metals in the centre. As a preliminary result, we found that the central metal ions play an important role in the interaction between DNA and metal porphyrin. The CD spectra revealed different behaviours of the porphyrins ranging from a side-on coordination, an intercalation or a partial intercalation of the porphyrin system into the DNA. To make this system more specific towards Z-DNA recognition, we are developing a system based on a porphyrin framework consisting of poly-(4-N-methylpyridinium)porpyhrins with a $3 \mathrm{~d}$ central metal and inorganic linkers in between the porphyrins. Taken into account the specific properties of Z-DNA like rigidity, base-tobase distance, coordination sites and sequence specific properties, we expect our unique system to recognize Z-DNA in a side-on coordination.

\section{Acknowledgements}

We would like to thank the University of Zürich, the Forschungskredit of the University of Zürich, the ETH Zürich and the Swiss National Science Foundation for financial support. We further thank Prof. Dr. Roger Alberto and his group as well as Dr. George Mitrikas for helpful discussions.

Received: January 13, 2009

[1] 'Nucleic Acid-Metal Ion Interactions', Ed. N. V. Hud, Royal Chemical Society, 2009, p. 433.

[2] E. Freisinger, R. K. O. Sigel, Coord. Chem. Rev. 2007, 251, 1834

[3] A. Rich, S. Zhang, Nat. Rev. Genet. 2003, 4 566.

[4] B. Spingler, C. Da Pieve, Dalton Trans. 2005, 1637.

[5] B. Spingler, F. Zobi, P. M. Antoni, A. MedinaMolner, R. Alberto, Chimia 2005, 59, 826.

[6] H.-C. Shih, N. Tang, C. J. Burrows, S. E. Rokita, J. Am. Chem. Soc. 1998, 120, 3284.

[7] C. Da Pieve, A. Medina-Molner, B. Spingler, Synthesis 2007, 679.

[8] C. Da Pieve, PhD Thesis, University of Zürich, 2005.

[9] T. Kan, A. Fujiwara, H. Kobayashi, T. Fukuyama, Tetrahedron 2002, 58, 6267.

[10] R. J. DeVita, M. T. Goulet, M. J. Wyvratt, M. H. Fisher, J. L. Lo, Y. T. Yang, K. Cheng, R. G. Smith, Bioorg. Med. Chem. Lett. 1999, 9, 2621.

[11] C. Agami, F. Couty, Tetrahedron 2002, 58, 2701

[12] C. A. Olsen, M. R. Jorgensen, M. Witt, I. R. Mellor, P. N. R. Usherwood, J. W. Jaroszewski, H. Franzyk, Eur. J. Org. Chem. 2003, 3288.

[13] A. Medina-Molner, PhD Thesis, University of Zürich, 2007.

[14] A. Medina-Molner, O. Blacque, B. Spingler, Org. Lett. 2007, 9, 4829.

[15] M. G. Santangelo, A. Medina-Molner, A Schweiger, G. Mitrikas, B. Spingler, J. Biol. Inorg. Chem. 2007, 12, 767.

[16] P. M. Schosseler, B. Wehrli, A. Schweiger Inorg. Chem. 1997, 36, 4490

[17] E. Sletten, B. Lie, Acta Cryst. 1976, B32, 3301.

[18] H. Sigel, B. Song, Met. Ions Biol. Syst. 1996 32,135 .

[19] N. Veldman, A. L. Spek, G. Tabbi, W. L. Driessen, J. Reedijk, Acta Cryst. 1996, C52, 2698.

[20] B. Spingler, P. M. Antoni, Chem. Eur. J. 2007, 13,6617

[21] M. Balaz, M. De Napoli, A. E. Holmes, A. Mammana, K. Nakanishi, N. Berova, R. Purrello, Angew. Chem., Int. Ed. 2005, 44, 4006. 